quency conversion and possibly to supercontinuum generation, increasing the possibility to design nonlinear fibers for dedicated applications and potentially enabling the generation of single-cycle optical pulses in the visible.

IULIA MUNTELE

\section{Magnetostatic Energy May Explain Unusual Ferromagnetic Transition in Erbium}

Erbium metal exhibits unusual behavior at the ferromagnetic transition. The firstorder transition between ferromagnetic $(\mathrm{F})$ and antiferromagnetic (AF) phases near $19 \mathrm{~K}$ demonstrates superheating and supercooling as well as an unusual mechanism behind the phase change. In material with small amounts of impurity, the phase transition is resolved into at least four subtransitions apparently connected with metastable phases occurring within a range of about 2 K. C.S. Durfee and C.P. Flynn at the University of Illinois at Urbana-Champaign account for this phenomenon in terms of magnetostatics which favors transitions through ferrimagnetic intermediaries.

As reported in the July 30 issue of Physical Review Letters, some features of the ferromagnetic nucleation in erbium can be explained by conventional methods. However, the researchers determined that the magnetostatic self-energy of the transformed nucleation droplet is enough to supercool the droplet to more than an order of magnitude greater than the observed $1 \mathrm{~K}$. They report that this behavior indicates that nucleation of the erbium phase cannot occur by the normal process of spherical droplets. The researchers instead propose the use of spheroidal droplets of large eccentricity. In spite of the increased surface area (by $\sim 200 \%$ ) of the spheroidal droplet over that of the spherical droplet, the magnetostatic self-energy is greatly diminished, reducing the barrier to nucleation. These anisotropic shapes represent the preferred path for droplet growth during the nucleation process. The researchers found that for $1 \mathrm{~K}$ supercooling, the length of the critical droplet along its major axis is now an order of magnitude larger than expected; however, the magnetostatic energy is still far too big and requires an impossibly large supercooling.

The researchers believe the answer lies in the metastable states accessed during the F-to-AF phase transition and consider ferrimagnetic intermediate (FI) phases, with 2, 4, or 6 spins aligned out of every 8 . They believe this explanation offers a low energy route between AF ( 0 excess spins) and $F$ (all 8 spins aligned) and that magnetostatics again plays a critical part. For the first ferrimagnetic phase the magnetostatic energy is reduced by a factor of 16 with a spheroidal droplet radius that has a surface energy $70 \%$ greater than a spherical nucleus. The barrier to nucleation for this FI droplet is now comparable with that of a spherical droplet in which the magnetostatic contribution is neglected. The researchers suggest that this explains the four-stage magnetic transition that has been observed.

JENNIFER BURRIS

\section{Volatile Metal Alkylamides Serve as Precursors for Vapor Deposition of High-Dielectric- Constant Gate Insulator Materials}

The speed and capacity of computers have been doubling every year or two, following Moore's law. These improvements in the power of microelectronics have been achieved largely by shrinking their dimensions. The smallest components in circuits are gate insulators,

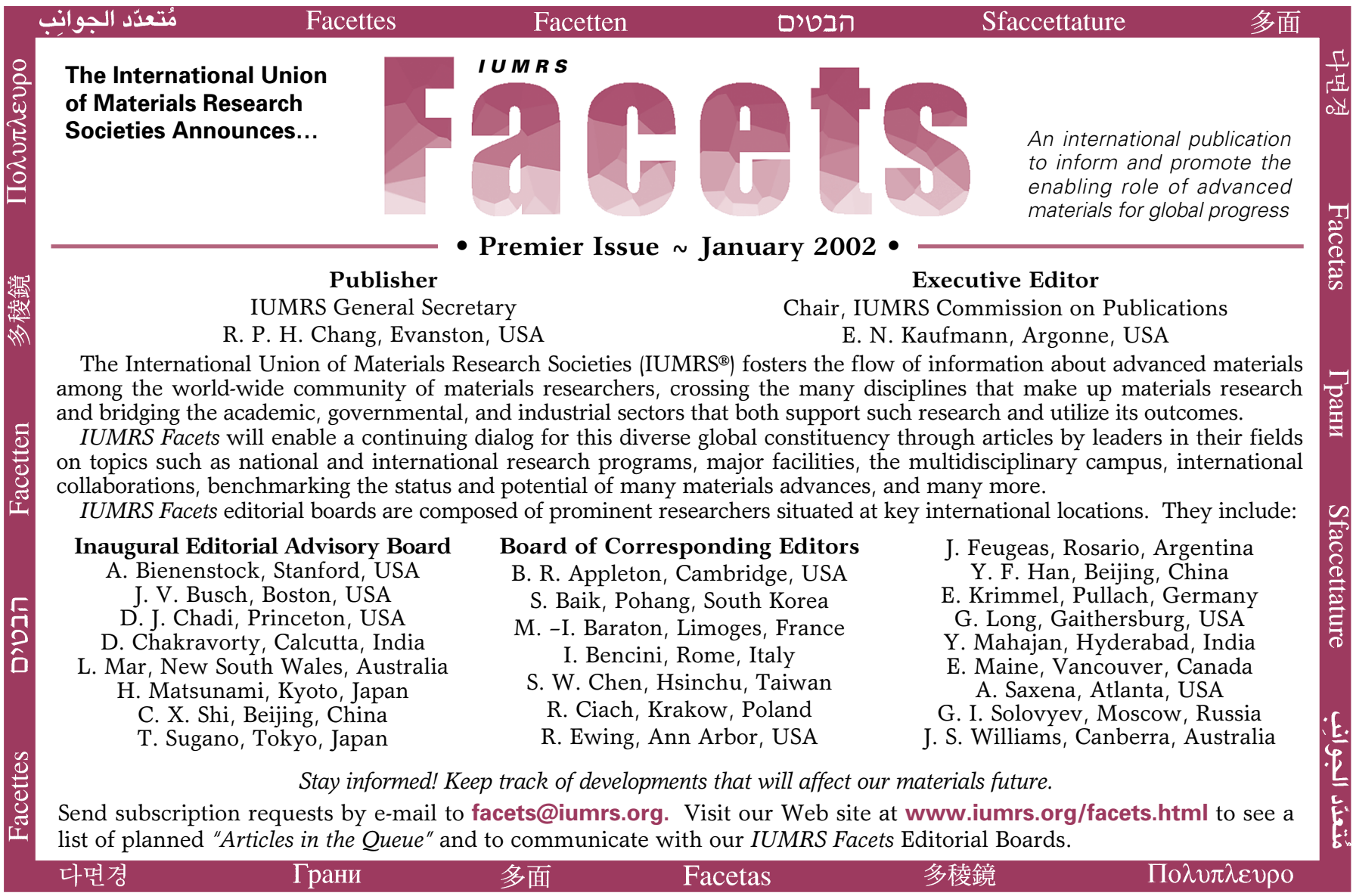

